\title{
Flore endemique, rare et menacée de l'Atlas tellien occidental de Tiaret (Algerie)
}

\author{
Mohamed Djamel Miara1, Mohammed Ait Hammou', Khellaf Rebbas ${ }^{2}$ \& Hamdi Bendif ${ }^{2}$ \\ ${ }^{1}$ Département de Biologie. Faculté SNV. Université Ibn Khaldoun, Tiaret. Algerie. 14000 DZ. \\ ${ }^{2}$ Département SNV. Faculté des sciences. Université de Msila. Algerie. 28000DZ.
}

\section{Correspondencia}

M.D. Miara

e-mail: miara14130@yahoo.fr

Recibido: 30 noviembre 2017

Aceptado: 21 diciembre 2017

Publicado on-line: diciembre 2017

\begin{abstract}
Resume
Cette étude vise la connaissance et la recherche des plantes endémiques, rares et menacées de liatlas tellien occidental de Tiaret. Les recherches ont été guidées par les données existantes dans la littérature afin de retrouver les plantes endémiques et rares citées pour la région de Tiaret. Nous avons exploité nos données de terrain (287 relevés floristiques) ainsi que dsautres recherches ciblées afin de localiser les taxons endémiques. Cela nous a permis d`identifier 32 plantes endémiques ( 7 endémiques strictes Algérie; 11 Algérie-Maroc; 4 Algérie-Tunisie; 10 Afrique du Nord). Les recherches ont permis également de reconnaitre 36 taxons rares, 4 Trigger species et 17 taxons menacées (protégés). Plusieurs taxons cités dans la bibliographie pour la région n'ont pas été retrouvés ce qui laisse à s'interroger quant à lıétat de conservation et l>existence de ces taxons dans un environnement très fortement perturbé par lsaction anthropique dévastatrice.
\end{abstract}

Mots clés: Algérie, endémiques, espèces protégés, espèces rares, Tell, Tiaret, Trigger species.

\section{Abstract}

Endemic, rare and threatered flora of the western tellien Atlas of Tiaret (Algeria

This study is aims at the knowledge and research of endemic, rare and endangered plants of the Western Tell Atlas of Tiaret. The research was guided by data existing in the literature to find the endemic and rare plants cited for the region of Tiaret. We used our field data (287 floristic records) as well as other targeted research to locate endemic taxa. This allowed us to identify 32 endemic plants (7 strict endemic of Algeria, 11 of Algeria-Morocco, 4 of Algeria-Tunisia, 10 of North Africa). The research also identified 36 rare taxa, 4 Trigger species and 17 threatened (protected) taxa. Several taxa listed in the bibliography for the region have not been found, which raises questions about the conservation status and the existence of these taxa in an environment that is highly disturbed by devastating human activity.

\section{Introduction}

A l'exception des dernières grandes régions sauvages encore préservées (forêts vierges équatoriales, dont l'Amazonie), près de la moitié du reste de la biodiversité mondiale se concentre au sein de 34 " points chauds ", ou hotspots (Myers, 2003). Ces points chauds de biodiversité dont l'intérêt biologique est avéré sont essentiellement caractérisés par leur diversité spécifique ainsi que de leurs taux d'endémisme élevés (Myers, 1990).

L'Algérie présente une richesse floristique remarquable qui est directement liée à sa diversité ecosystémique et paysagère. Sa flore est estimée à 3994 taxons, le nombre de ceux endémiques est de 464 (387espèces, 53 sous-espèces et 24 
variétés), soit11.61 \% des plantes vasculaires algériennes (Yahi \& Benhouhou in Radford et al., 2011).

Les taxons rares en Algérie varient quant à eux selon les secteurs biogéographiques. D'après Véla \& Benhouhou (2007), les taxons plus ou moins rares en Algérie (avec une abondance allant de AR au RRR au sens de Quézel \& Santa(1962-63)) sont au nombre de 1818 taxons à travers tous les secteurs biogéographiques du pays. Ces indices qui sont indépendants à la notion d'endémisme sont en effet à actualiser à la lumière des données récentes.

Le secteur de l'Atlas tellien occidental par sa position géographique, sa diversité paysagère et phytocénotique, pourrait constituer un refuge des plantes endémiques, rares ou menacées souvent vulnérables par rapport aux contraintes écologiques et anthropiques.

En effet, les modifications que subissent à la fois les écosystèmes et les paysages en Algérie constituent une réelle contrainte au maintien de cette diversité floristique et notamment les plantes les plus vulnérables.

La recherche et la protection de ces plantes s'impose ainsi comme une urgence afin de pouvoir sauvegarder ce patrimoine biologique d'intérêt majeur.

Cette étude aborde cette problématique au niveau de la région de Tiaret faisant partie du sous-secteur de l'Atlas tellien oranais (O3) dont les conditions écologiques et anthropiques actuelles laissent à s'interroger quant à la situation de ces taxons. Elle pourra également apporter de nouveaux éléments de connaissance sur la flore rare et endémique à l'échelle du pays.

Les recherches consistent à identifier et localiser ces plantes au niveau régional (recherches de terrain), puis de détecter les éventuels changements qu'ont subis ces taxons notamment sur le plan biogéographique depuis les récentes révisions (actualisation des données).

Globalement, notre étude régionale a pour but de préciser et vérifier les connaissances sur cette flore en vue d'en envisager une meilleure protection et conservation. Cette conservation concernera ainsi, les habitats naturels de ces plantes qui demeurent actuellement sans statut légal de zones protégées.

\section{La zone d'etude}

La région de l'Atlas tellien occidental de Tiaret se situe au nord-ouest de l'Algérie occupant de vastes espaces montagneux forestiers (fig.1). Elle est essentiellement représentée au niveau de la partie nord du territoire de la wilaya de Tiaret. Cela correspond sur le plan biogéographique aux zones naturelles des collines de Tiaret ainsi que les monts de Frenda (Duvignaud, 1992). La région bénéficie d'un climat semi-aride dominant dans la variante fraiche (Miara et al., 2013).

Cette région est couverte par une végétation préforestiere constituée par différentes séries de végétation dont les plus dominantes sont celles du Chêne vert et du pin d'Alep (Miara, 2017). Les sols sont essentiellement calcaires sur la partie des

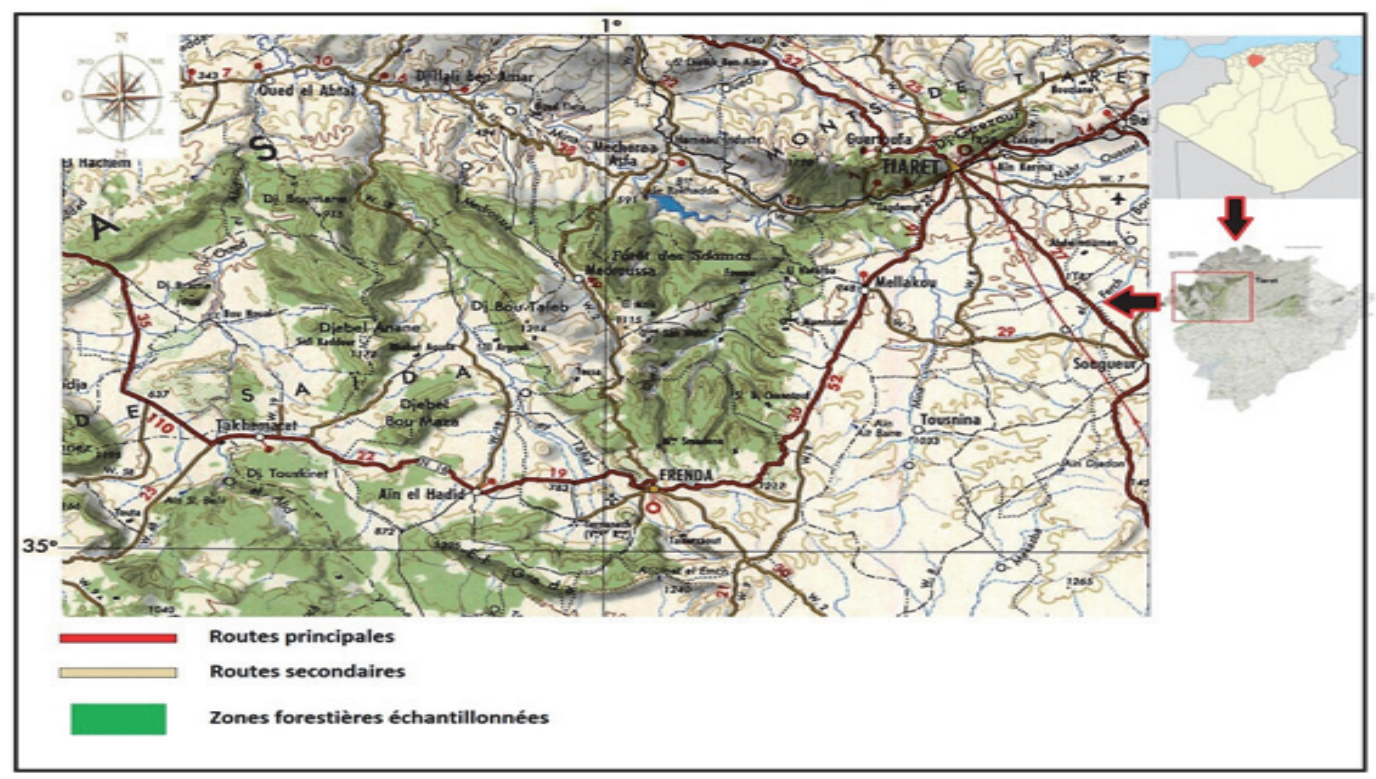

Figure 1. Situation géographique de la région de Tiaret (Extrait de la carte d'Algérie, feuille de Tiaret, 1/200 000). Figure 1. Location of the region of Tiaret (Extract from the map of Algeria, sheet of Tiaret, 1/200 000). 
monts de Frenda alors qu'au niveau des monts de Tiaret, c'est plutôt les sols sableux décarbonatés qui dominent.

\section{Materiel et methodes}

La méthodologie adoptée ici se résume en une synthèse des données bibliographiques et de terrains. Ces derniers ont été récoltés suivant deux types d'échantillonnage différents.

D'abord, nous avons utilisé les données de terrain recueillis à travers les 287 relevés phytoécologiques réalisés dans différents points du secteur Atlasique de Tiaret (fig.2) selon un échantillonnage stratifié (Miara, 2017). Ces données nous ont permis d'identifier 566 taxons dont 29 endémiques relevant des quatre catégories d'endémisme (Algériennes strictes, Algéro-Marocaines, Algéro-Tunisiennes et NordAfricaines). L'analyse de ces données nous a aussi permis d'extraire les espèces rares (au sens de Quézel \& Santa., 1962-63) ainsi que les taxons menacées (après consultation de la liste des espèces protégées en Algérie- décret exécutif n ${ }^{\circ} 12-03$ du 10 safar 1433 correspondant au 4 janvier 2012 fixant la liste des espèces végétales non cultivées protégées).

Par la suite, nous avons procédé à des sorties de terrain de manière ciblée afin de rechercher certains taxons rares et/ou endémiques d'après la littérature.

Les zones ainsi visitées sont en effet les régions de présence potentielle de ces taxons d'après les données bibliographiques (flores anciennes, herbiers, articles anciens...etc.). Mais aussi, certaines zones suspectes comme les points inaccessibles et ou dangereux d'accès ou même celles que nous n'avons pas pu visiter lors de la réalisation des relevés. Ainsi, le choix de ces zones de recherches a été apprécié d'une manière plus ou moins subjective-dirigée. Sur le terrain, les spécimens d'herbier ont été récoltés ainsi que des prises de photos notamment pour les plantes endémiques, rares et menacées. Les spécimens d'herbier collectés ont été identifiés puis déposés au niveau de l'herbier du département de biologie de l'université de Tiaret.

L'identification botanique a été faite à l'aide des documents suivants: Battandier \& Trabut (1888-1890), Battandier \& Trabut (1895), Maire (1952-1987) et Quézel \& Santa (1962-63). Ces documents nous ont servi pour la recherche et la détermination botanique de nos taxons ainsi que pour la comparaison de leurs aires géographiques respectives. Pour l'actualisation des données sur la nomenclature, la taxonomie et la chorologie, nous avons consulté El Oualidi et al. (2012) et Dobignard \& Chatelain (2010-11-12-13) ainsi que la base de données «EuroMed plant database» disponible sur le web (www.emplantbase.org).

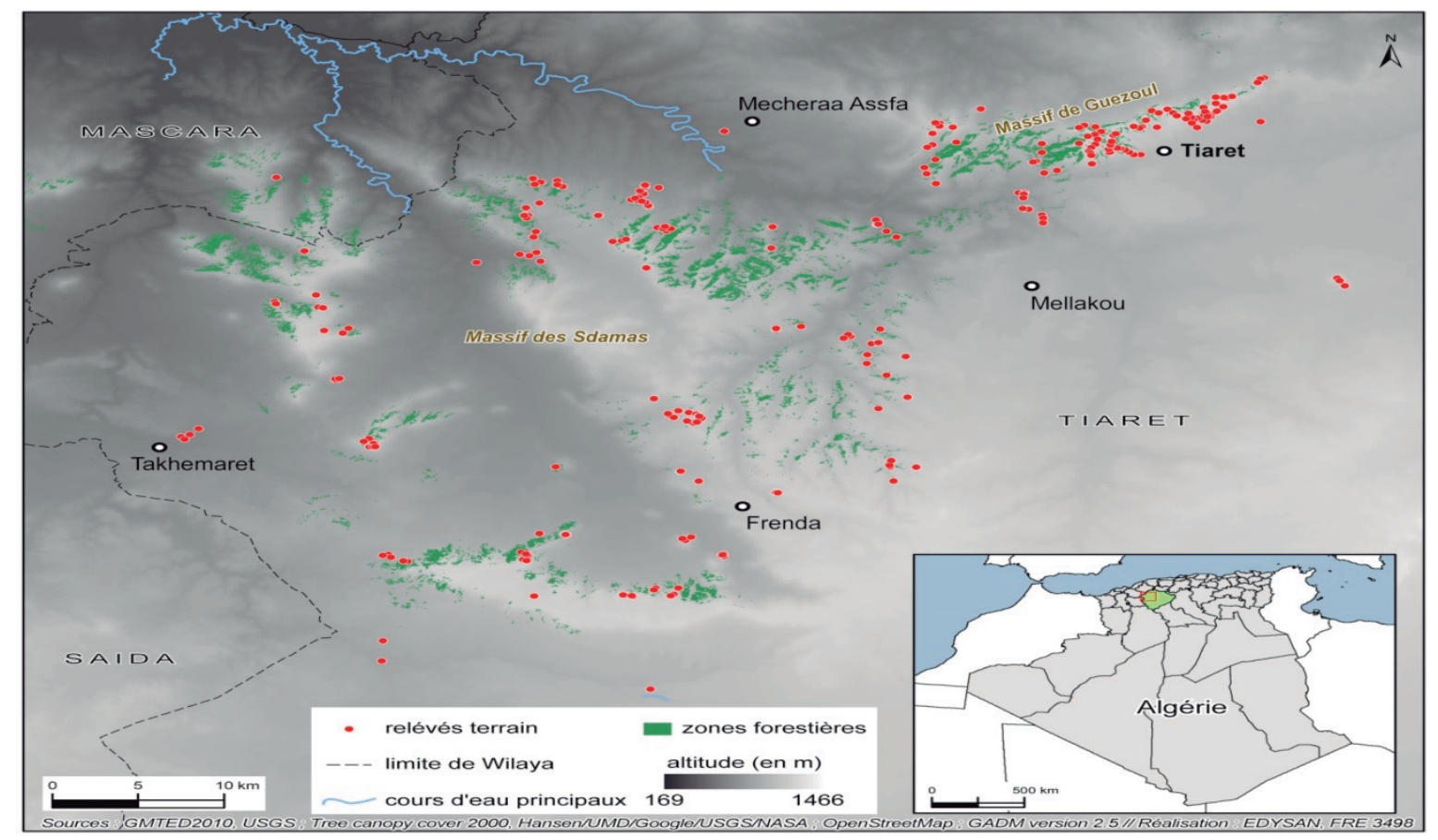

Figure 2. Carte de localisation des relevés floristiques réalisés à travers l'Atlas tellien occidental de Tiaret (Miara, 2017). Figure 2. Location map of floristic surveys carried out through the Western Telli Atlas of Tiaret (Miara, 2017) 


\begin{tabular}{|c|c|c|}
\hline $\begin{array}{l}\text { Espèces endémiques } \\
(Q \text { \& S (62-63) }\end{array}$ & $\begin{array}{l}\text { Chorologie } \\
\text { Q\&S (62-63) }\end{array}$ & $\begin{array}{l}\text { Chorologie } \\
\text { D \& Ch (10-13) }\end{array}$ \\
\hline Adenocarpus faurei Maire & End Alg & End Alg \\
\hline Armeria ebracteata Pomel & End Mar E Alg & End Alg-Mar \\
\hline Astragalus gryphus Coss. \& Durieu ex Bunge & End W.N.A & End Alg-Mar \\
\hline Centaurea obtusiloba & End Alg & End Alg \\
\hline Cerastium atlanticum Durieu & End Alg & End Alg-Mar-Tun \\
\hline Clypeola cyclodontea Delile & End N.A & Alg-Mar-Esp \\
\hline $\begin{array}{l}\text { Cynosurus elegans ssp aurasiacus = Cynosurus elegans Desf. } \\
\text { subsp. elegans }\end{array}$ & End Alg & End Alg-Tun \\
\hline Evax crocidion $=$ Filago crocidion $($ Pomel) Chrtek \& Holub & End Alg. Mar & End Alg-Mar \\
\hline Nepeta algeriensis de Noé & End Alg & End Alg-Tun \\
\hline Papaver malviflorum Doum. & End Alg & End Alg-Mar \\
\hline Ranunculus batrachioides Pomel & End Alg & Alg-Mar-Esp \\
\hline Spergularia tenuifolia Pomel & End Alg & End Alg-Mar \\
\hline Trisetaria nitida (Desf.) Maire & End Alg & End End Alg \\
\hline
\end{tabular}

Table 1. Espèces endémiques signalées pour la région de Tiaret d'après Quézel \& Santa (1962). Chorologie actualisée selon Dobignard \& Chatelain (2010-11-12-13). Table 1. Endemic species reported for the region of Tiaret after Quézel \& Santa (1962). Chorology updated according to Dobignard \& Chatelain (2010-11-12-13).

\begin{tabular}{|l|c|c|}
\hline Catégories d'endémisme & Total (Q \& S 62-63) & Total D \&Ch (10-13) \\
\hline Endemiques & 12 & 10 \\
\hline Endemiques et rares & 10 & 2 \\
\hline End Alg & 8 & 5 \\
\hline End Alg-Mar & 2 & 2 \\
\hline End Alg-Tun & 0 & 1 \\
\hline End Alg-Mar-Tun & 0 & 0 \\
\hline End (Alg-Mar-Tun-Lyb) & 1 & 2 \\
\hline $\begin{array}{l}\text { Afrique nord occidentale-Europe sud-occidentale (Alg-Mar- } \\
\text { Tun-Esp-Port-It) }\end{array}$ & 0 & 2 \\
\hline
\end{tabular}

Table 2. Tableau récapitulatif des statuts des taxons endémiques de la région de Tiaret d'après la bibliographie. Chorologie actualisée selon Dobignard \& Chatelain (2010-13). Table 2. Table summarizing the status of endemic taxa in the region of Tiaret according to the bibliography. Chorology updated according to Dobignard \& Chatelain (2010-13).

Nous avons enfin combiné les données des relevés floristiques à celles obtenues suites aux sorties de recherches des plantes endémiques et I ou rares pour pouvoir présenter au final des listes des plantes endémiques, rares et menacées.

\section{Resultats et discussion}

\section{Les plantes endemiques citees pour la region de tiaret (bibliographie)}

Nos recherches au niveau des massifs telliens de Tiaret ont été d'abord guidées par les données de la littérature sur les taxons endémiques régionaux. Plusieurs taxons endémiques signalés d'après les flores d'Algerie (Battandier \& Trabut,1888-1890, Battandier \& Trabut, 1895, Maire, 1952-1987 et Quézel \& Santa, 196263) au niveau de certaines régions de Tiaret ne relevant pas de nos périmètres d'investigation se situant dans les parcours steppiques n'ont pas été recherchés. C'est le cas des taxons suivants: Spergularia tenuifolia Pomel, Astragalus gryphus Coss. \& Durieu ex Bunge, Armeria ebracteata Pomel, Evax crocidion (= Filago crocidion (Pomel) Chrtek \& Holub), Cerastium atlanticum Durieu et Clypeola cyclodontea Delile. Or, certains d'autres (Adenocarpus faurei, Trisetaria nitida, Centaurea obtusiloba) n'ont pas été retrouvés dans leurs 


\begin{tabular}{|c|c|c|c|}
\hline Espèces endémiques & $\begin{array}{l}\text { Chorologie Q\&S } \\
\text { (62) }\end{array}$ & Chorologie Q\&S (62) & Distribution régionale \\
\hline Crupina intermedia (Mutel) Walp. & End Alg-Mar & Alg-Mar-Ital & Dj. Sidi Abed (Sougueur) \\
\hline $\begin{array}{l}\text { Fumana fontanesii Pomel = Fumana } \\
\text { calycina }\end{array}$ & End Alg-Mar & $\mathrm{NA}+\mathrm{Esp}$ & $\begin{array}{l}\text { Tagdempt, Laawidja, Sidi Bakhti } \\
\text { (Mechraa sfa), Tafza(Ain El Hdid), } \\
\text { Dj. Ghzalla (Mechraa sfa). }\end{array}$ \\
\hline Galium tunetanum Lam. & End N.A & Alg-Mar-Tun-It-Esp & Dj. Boumaaza (Ain El Hdid) \\
\hline $\begin{array}{l}\text { Glossopappus macrotus (Durieu) Briq. \& } \\
\text { Cavill. }\end{array}$ & End Alg & Alg-Mar-Tun-Esp-Port & $\begin{array}{l}\text { Dj. Hlayllia (Guertoufa), Mechraa } \\
\text { sfa, Fontaine gibbon (Dj. Guezoul), } \\
\text { Dj Ghzalla (Mechraa sfa) }\end{array}$ \\
\hline Helianthemum polyanthum (Desf.) Pers. & End Alg-Mar & Alg-Mar-Esp & $\begin{array}{l}\text { Tagdempt, Dj. Boumaaza (Ain El } \\
\text { Hdid), Tmixi (Takhmaret) }\end{array}$ \\
\hline $\begin{array}{l}\text { Patzkea patula (Desf.) H. Scholz = Festuca } \\
\text { triflora }\end{array}$ & End Alg-Mar & Alg/Mar/Esp & Tahamamet (Frenda) \\
\hline $\begin{array}{l}\text { Scrophularia laevigata Vahl = Scrofularia } \\
\text { laevigata ssp. simplicifolia }\end{array}$ & End N.A & Alg-Mar-Tun-Esp-Port & Belmorsli (Mechraa sfa) \\
\hline
\end{tabular}

Table 3. Changements chorologiques des taxons endémiques observés dans la région de Tiaret. Chorologie actualisée selon Dobignard \& Chatelain (2010-13). Table 3. Chorological changes of endemic taxa observed in the region of Tiaret. Chorology updated according to Dobignard \& Chatelain (2010-13).

périmètres biogéographiques dans lequels ils ont été signalés ce qui laisse à s'interroger sur leur état ou même leur existence actuelle.

Basée sur un specimen d'herbier de $\mathrm{Mr}$ Alphonce Faure (1865-1958), Adenocarpus faurei a été decrit par Dr. R. Maire en 1926 comme une endemique des vallons de l'oued sidi khaled sur les hauteurs de la ville de Tiaret. Cet arbuste inerme fleurant penadant le mois de juillet n'a jamais été revue depuis. Une étude a été lancée a la recherche de cette plante pendant 5 années n'a malheureusement pas été fructueuse (Miara et al, 2018). II en est vraissemblablement de meme pour Trisetaria nitida, Centaurea obtusiloba qui ont été decrits pour la premiere fois dans les monts de Tiaret mais qui n'ont jamais été revues depuis.

L'actualisation des données chorologiques des espèces endémiques montre que certains d'entre elles ne sont désormais plus endémiques suite à des découvertes dans les pays d'Europe méditerranéenne comme l'Espagne ou l'Italie (tab.1). C'est en effet le cas de Ranunculus batrachioides ou de Clypeola cyclodontea.

C'est ainsi que le nombre des endémiques algériennes citées pour Tiaret a diminué de 12 à 10 taxons contre une augmentation de ceux de statut Algéro-Marocains et Algéro-Tunisiens (tab. 2).

De ce fait, la mise à jour nomenclaturale des taxons dans les études floristiques, bien que d'importance indéniable, elle doit être toujours suivie par une autre des données chorologiques notamment concernant les taxons endémiques dont nous avons constaté des changements significatifs.

\section{Les plantes endemiques observees dans la region de tiaret}

Les recherches menées à travers cette étude nous ont permis de comptabiliser 36 plantes endémiques que nous avons identifiées dans la région. Ce nombre pouvant être jugé important, représente environ $38 \%$ de la flore endémique du secteur biogéographique (O3) qui est de 94 espèces (Véla \& Benhouhou, 2007). Ce nombre représente également près de $9 \%$ de la flore endémique totale du pays qui est de 406 espèces selon ces mêmes auteurs.

\section{Chorologie des taxons}

Sur le plan chorologique, nous avons constaté que plusieurs taxons ont changé de statut.

En effet, nous avons identifié des taxons endémiques sensu (Quézel \& Santa, 196263) qui ne sont désormais plus endémiques d'après Dobignard \& Chetelain (2010-11-1213). Ces taxons sont : Crupina intermedia, Fumana fontanesii Pomel (= Fumana calycina), Galium tunetanum, Glossopappus macrotus, Helianthemum polyanthum, Hypecoum littorale Wulfen. (= Hypecoum geslini Coss. et Kral.), Patzkea patula (Desf.) H. Scholz (= Festuca triflora) et Scrophularia laevigata Vahl (= Scrofularia laevigata ssp. simplicifolia) (tab.3).

Certainsd'autresontchangéd'aired'endémisme tout en gardant leurs statut d'endémiques comme Linum tenue subsp. munbyanum endémique nordAfricain qui est devenue endémique Algérie-Maroc.

Gagea algeriensis endémique algérienne est devenue endémiques Algéro-Marocaine. Genista 


\begin{tabular}{|c|c|c|c|}
\hline Especes endemiques & $\begin{array}{c}\text { Chorologie Q\&S } \\
\mathbf{( 6 2 )}\end{array}$ & $\begin{array}{c}\text { Chorologie Q\&S } \\
\text { (62) }\end{array}$ & Distribution régionale \\
\hline $\begin{array}{c}\text { Coleostephus multicaulis (Desf.) Durieu = Chry- } \\
\text { santhemum multicaule Desf. }\end{array}$ & End Alg & Fontaine gibbon (Dj. Guezoul) \\
\hline Erodium medeense Batt. & End Alg & End Alg & Guertoufa \\
\hline Centaurea phaeolepis Coss. & End Alg & End Alg & Guertoufa \\
\hline Otocarpus virgatus Durieu & End Alg & End Alg & Dj. Boughachwa (Frenda) \\
\hline Teucrium $\begin{array}{c}\text { aureiforme Pomel = Teucrium polium } \\
\text { ssp. aureiforme }\end{array}$ & - & End Alg & Bekhadda (Mechraasfa) \\
\hline Thymus guyonii de Noé & End Alg & End Alg & Dj. Hlayllia (Guertoufa) \\
\hline Thymus lanceolatus Desf.
\end{tabular}

Table 4. Les endémiques stricts-Algérie retrouvés dans la région de Tiaret. Table 4. Strict endemics-Algeria found in Tiaret region.

\begin{tabular}{|c|c|c|c|}
\hline Espèces endémiques & Chorologie Q\&S (62) & $\begin{array}{c}\text { Chorologie Q\&S } \\
(62)\end{array}$ & Distribution régionale \\
\hline Centaurea oranensis Greuter \& M.V.Agab. & End Alg-Mar & End Alg-Mar & Dj. Sidi bed (Sougueur) \\
\hline Cistus munbyi Pomel $=$ Cistus sericeus & End Alg-Mar & End Alg-Mar & Dj. Boumaaza, Dj. Boutaleb \\
\hline Cordylocarpus muricatus Desf. & End Alg-Mar & End Alg-Mar & Dj. Sidi Abed (Sougueur) \\
\hline Gagea algeriensis Chabert & End Alg & End Alg-Mar & Dj. Ghzalla (Mechraa sfa) \\
\hline $\begin{array}{l}\text { Genista cephalantha Spach. = Genista } \\
\text { cephalantha Spach. ssp. demnatensis. }\end{array}$ & End $\mathrm{N}-\mathrm{A}$ & End Alg-Mar & Laawidja (Mechraa sfa) \\
\hline $\begin{array}{c}\text { Linum tenue subsp. munbyanum (Boiss. \& } \\
\text { Reut.) Batt. }\end{array}$ & End N.A & End Alg-Mar & $\begin{array}{l}\text { Dj. Guezoul, Mechraa sfa, } \\
\text { Frenda, Dj. Boumaaza (Ain El } \\
\text { Hdid) }\end{array}$ \\
\hline Romulea numidica Jord. \& Fourr & End Alg-Mar & End Alg-Mar & Dj. Guezoul \\
\hline Salvia algeriensis Desf. & End Alg-Mar & End Alg-Mar & Dj. Guezoul \\
\hline Sideritis guyoniana Boiss. \& Reut. & - & End Alg-Mar & Dj. Tmixi (Takhmaret) \\
\hline $\begin{array}{l}\text { Thymus munbyanus Boiss. \& Reut. subsp. } \\
\text { munbyanus = Thymus ciliatus ssp. munbyanus }\end{array}$ & End N.A & End Alg-Mar & Partout \\
\hline $\begin{array}{c}\text { Verbascum faurei (Murb.) Hub.-Mor. }=\text { Celsia } \\
\text { faurei }\end{array}$ & End Alg & End Alg-Mar & Dj. M’khatria (Guezoul) \\
\hline $\begin{array}{l}\text { Thymus pallescens de Noé }=\text { Thymus } \\
\text { fontanesii }\end{array}$ & $\begin{array}{l}\text { End Alg-Tun (El Oualidi et } \\
\text { al, 2012) }\end{array}$ & End Alg-Mar & Dj. Guezoul \\
\hline
\end{tabular}

Table 5. Les endémiques Algérie-Maroc retrouvés dans la région de Tiaret. Table 5. Algeria-Morocco endemics found in the region of Tiaret.

cephalantha Spach. (= Genista cephalantha Spach. ssp. demnatensis) et Thymus munbyanus Boiss. \& Reut. subsp. munbyanus (= Thymus ciliatus ssp. munbyanus) endémiques nord-Africaines qui sont devenues endémiques Algéro-Marocaines.

Hedysarum pallidum endémique AlgéroTunisienne qui est devenue endémique nord-Africaine. Linum corymbiferum Desf. subsp. corymbiferum endémique nord-Africaine qui est devenue endémique Algéro-Tunisienne.

Rosmarinus eriocalyx Jord. \& Fourr. (= Rosmarinus tournefortii) endémique algérienne qui est devenue endémique (Algérie-Maroc-Lybie).

Enfin les taxons qui ont conservé leurs statut d'endémisme sont : Centaurea oranensis Greuter \& M.V.Agab, Centaurea parviflora Desf., Centaurea phaeolepis Coss., Cistus munbyi Pomel = Cistus sericeus, Coleostephus multicaulis (Desf.) Durieu = Chrysanthemum multicaule, Cordylocarpus muricatus Desf., Delphinium balansae, Ebenus pinnata Aiton, Ephedra altissima Desf., Erodium medeense Batt., Genista tricuspidata Desf. subsp. tricuspidata, Helianthemum helianthemoides (Desf.) Grosser., Helianthemum ledifolium subsp. apertum (Pomel) Raynaud ex Greuter \& Burdet., Origanum vulgare subsp. glandulosum (Desf.) letsw. = Origanum glandulosum Desf., Otocarpus virgatus Durieu., Pancratium foetidum, 


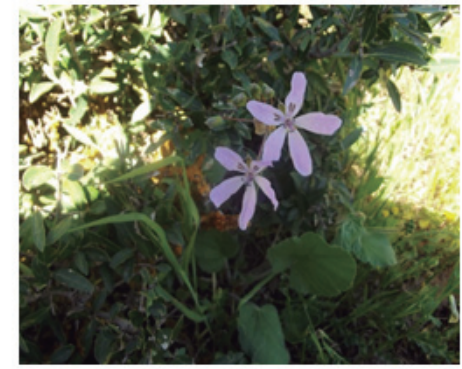

Erodium medeens

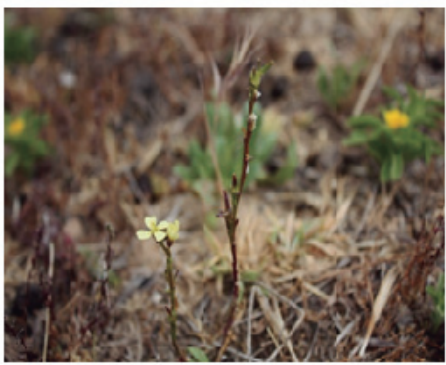

Otocarpus virgatus

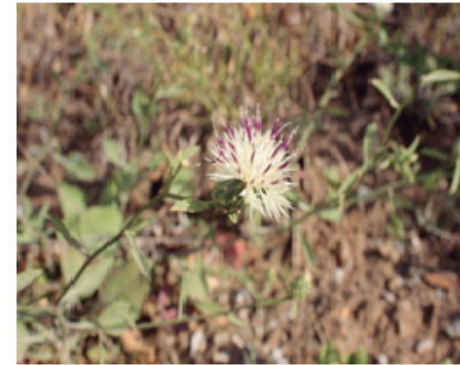

Centaurea phaelepis

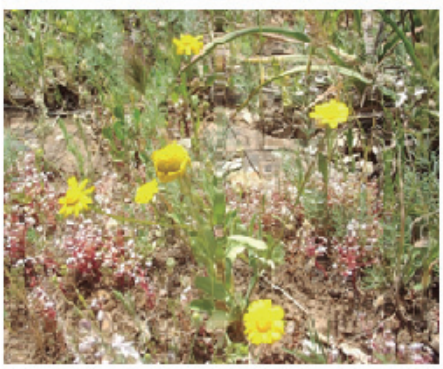

Coleostephus multicaulis

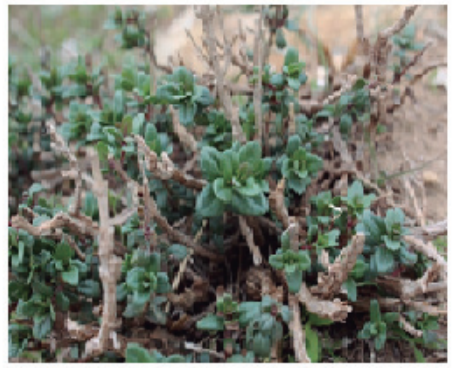

Thymus guyonii

Planche 1. Quelques endémiques strictes algériennes de la région de Tiaret (Cliché: Miara M D). Planche 1.Some strict Algerian endemic of the region of Tiaret (Photo: Miara M D).

Pistacia atlantica Desf., Romulea numidica Jord. \& Fourr., Salvia algeriensis Desf., Thymus algeriensis Boiss. \& Reut., Thymus guyonii de Noé., Thymus lanceolatus Desf.

Par ailleurs, nous signalons l'absence de l'espèce Cordylocarpus muricatus Desf. au niveau de la liste publiée par El Oualidi et al. (2012).

\section{Les endemiques algeriennes}

Les taxons endémiques stricts de l'Algérie que nous avons identifiés (tab 3-planche.1) sont de l'ordre de 7 taxons qui sont:

Centaurea phaeolepis Coss.qui est une espèce rare dans la région de Tiaret et de Bossuet à Belabess (Quézel et Santa, 1962-63);

Erodium medeense Batt. décrit par Battandier \& Trabut (1888-1890) a Médéa (Nador de Médéa, Ben chicao), ce taxon fait défaut dans la flore de Quézel \& Santa (1962-63). II a été par la suite confirmé comme endémique algérienne par Guittonneau (1972);

Otocarpus virgatus Durieu a été signalé par Trabut (1887) dans la région de Saida, puis par Maire (1965) à Saïda et Frenda et Tafaraoua. II a été récemment signalé à Djebel Boughachwa (Miara et al.,2014);

Thymus guyonii de Noé est une plante rare citée dans les flores d'Algérie uniquement pour le $\mathrm{H} 1$ etles régions semi-arides (Aflou, Djelfa, ...etc). Elle a été retrouvée dans la région de Mechraa Sfa près du barrage de Bekhadda ainsi qu'à Djebel Mkhatria au niveau du massif de Guezoul;

Coleostephus multicaulis (Desf.) Durieu, est une endémique très commune en Oranie $(\mathrm{O} 1$, O2 et O3) selon Quézel \&Santa (1962-63). Nous l'avons localisée au niveau des pelouses et clairières forestières du massif de Guezouldans les monts de Tiaret; 


\begin{tabular}{|c|c|c|c|}
\hline Espèces endémiques & $\begin{array}{c}\text { Chorologie } \\
\text { Q\&S (62) }\end{array}$ & $\begin{array}{c}\text { Chorologie } \\
\text { Q\&S (62) }\end{array}$ & Distribution régionale \\
\hline Centaurea parviflora Desf. & End Alg-Tun & End Alg-Tun & Massif de Guezoul \\
\hline $\begin{array}{c}\text { Linum corymbiferum Desf. subsp. corymbiferum. } \\
\begin{array}{c}\text { Origanum vulgare subsp. glandulosum (Desf.) letsw. } \\
\text { = Origanum glandulosum Desf.. }\end{array}\end{array}$ & End N-A & End Alg-Tun & $\begin{array}{c}\text { Dj. Boutaleb } \\
\text { (Mechraa sfa) }\end{array}$ \\
\hline
\end{tabular}

Table 6. Les endémiques Algérie-Tunisie retrouvés dans la région de Tiaret. Table 6. The Algeria-Tunisia endemics found in the region of Tiaret.

\begin{tabular}{|c|c|c|c|}
\hline Espèces endémiques & $\begin{array}{l}\text { Chorologie Q\&S } \\
\text { (62) }\end{array}$ & Chorologie Q\&S (62) & Distribution régionale \\
\hline Asphodelus acaulis Desf. & End N-A & End Alg-Mar-Tun & Dj. Guezoul \\
\hline Delphinium balansae Boiss. \& Reut. & End N.A & End Alg-Mar-Tun & Dj. Guezoul \\
\hline Ebenus pinnata Aiton & End N.A & End Alg-Mar-Tun-Lyb & $\begin{array}{l}\text { Mechraasfa, Frenda, } \\
\text { Lohou, Guezoul }\end{array}$ \\
\hline Ephedra altissima Desf. & End $\mathrm{N}-\mathrm{A}$ & End N-A & $\begin{array}{l}\text { Sidi Bakhti (Mechraa sfa), } \\
\text { Frenda }\end{array}$ \\
\hline $\begin{array}{l}\text { Genista tricuspidata Desf. subsp. tricuspidata } \\
=\text { Genista tricuspidata ssp. eu-tricuspidata }\end{array}$ & End N.A & End Alg-Mar-Tun & Partout \\
\hline Hedysarum pallidum Desf. & End Alg-Tun & End Alg-Mar-Tun & $\begin{array}{l}\text { Dj. Guezoul, Tafza (Ain El } \\
\text { Hdid) }\end{array}$ \\
\hline Helianthemum helianthemoides (Desf.) Grosser & End N.A & End Alg-Mar-Tun-Lyb & Dj. Guezoul \\
\hline $\begin{array}{c}\text { Helianthemum ledifolium subsp. apertum (Pomel) } \\
\text { Raynaud ex Greuter \& Burdet. = Helianthemum } \\
\text { apertum pommel }\end{array}$ & End $\mathrm{N}-\mathrm{A}$ & End Alg-Mar-Tun & Frenda \\
\hline $\begin{array}{l}\text { Hypecoum littorale Wulfen. = Hypecoum geslini } \\
\text { Coss. et Kral.. }\end{array}$ & End $\mathrm{N}-\mathrm{A}$ & Non End / Méd & Gaada (Frenda) \\
\hline Pancratium foetidum & End $\mathrm{N}-\mathrm{A}$ & End $\mathrm{N}-\mathrm{A}$ & Dj. Guezoul \\
\hline Pistacia atlantica Desf. & End N.A & Alg-Mar-Tun-Lyb & Sidi Wadeh (Tagdempt) \\
\hline $\begin{array}{l}\text { Rosmarinus eriocalyx Jord. \& Fourr. = } \\
\text { Rosmarinus tournefortii }\end{array}$ & End Alg & Alg-Mar-Lyb & $\begin{array}{l}\text { Sdamas (Mechraasfa, } \\
\text { Frenda, Lohou, Takhmaret, } \\
\text { Ain el Hdid) }\end{array}$ \\
\hline Stipa balansae H. Scholz & End N.A & Alg-Mar-Tun & $\begin{array}{l}\text { Tamelaht (Ain El Hdid), } \\
\text { Oued Fardja (Frenda) }\end{array}$ \\
\hline Thymus algeriensis Boiss. \& Reut. & End $\mathrm{N}-\mathrm{A}$ & End $\mathrm{N}-\mathrm{A}$ & Frenda \\
\hline
\end{tabular}

Table 7. Les endémiques Nord-Africaines retrouvés dans la région de Tiaret. Table 7. The endemic North Africans found in the region of Tiaret.

Thymus lanceolatus Desf. a été signalé de l'Oranie (O3) a l'ouest jusqu'à l'algérois (A2) à l'est mais aussi dans les régionsdes hauts plateaux intérieurs ( $\mathrm{H} 1$ et $\mathrm{H} 2)$. Cette plante a été retrouvée à Djebel Hlaylia au niveau du massif de Guezoul;

Teucrium aureiforme Pomel. est une plante citée dans le rang infra-spécifique par Quézel \& Santa (1962-63). Ce taxon figure dans Dobignard \& Chatelain (2010-13) comme une espèce endémique algérienne.

Comparé à des études semblables, notamment celles Medjahdi et al. (2009) dans les Traras de
Tlemcen ou encore celle de Hamel et al. (2013) dans l'Edough, le nombre des endémiques strictes présentes dans notre région pourrait être considéré comme important. II représente environ $15 \%$ de la flore endémique stricte du secteur $\mathrm{O} 3$ qui comporte 41 taxons (Véla \& Benhouhou, 2007).

\section{Les endemiques Algerie-Maroc}

Nous avons comptabilisé 11 espèces endémiques Algéro-Marocaines à travers notre région d'étude (tab. 5) dont certaines ont été photographiées sur le terrain (planche 2). 


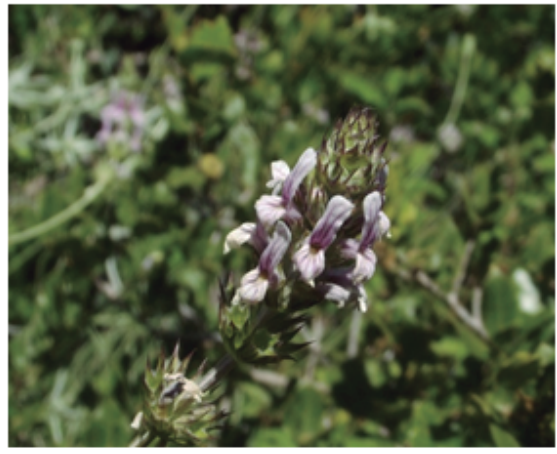

Sideritis guyoniana

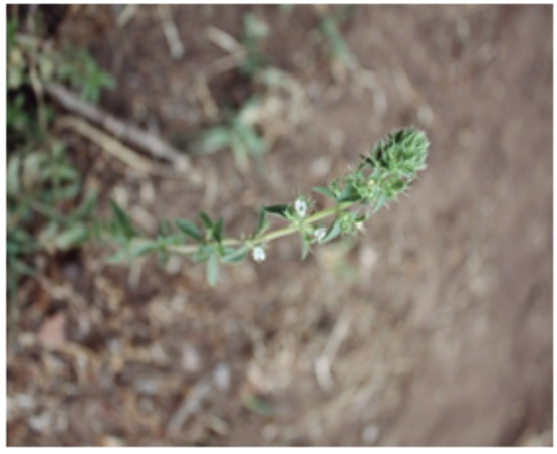

Thymus pallescens

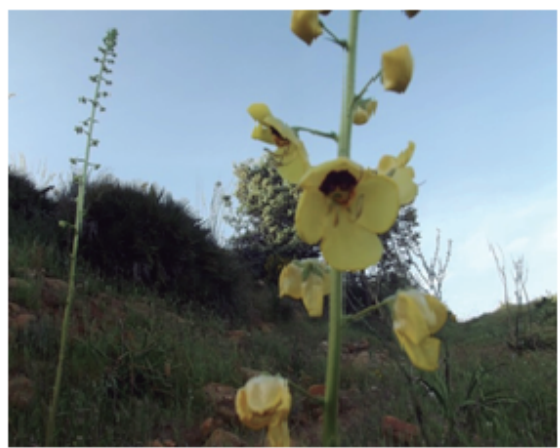

Verbascum faurei

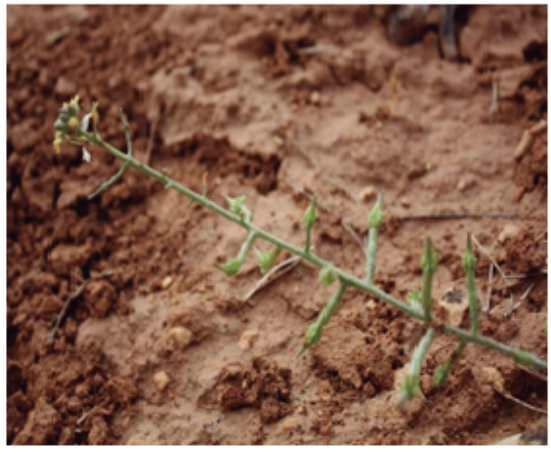

Cordylocarpus muricatus

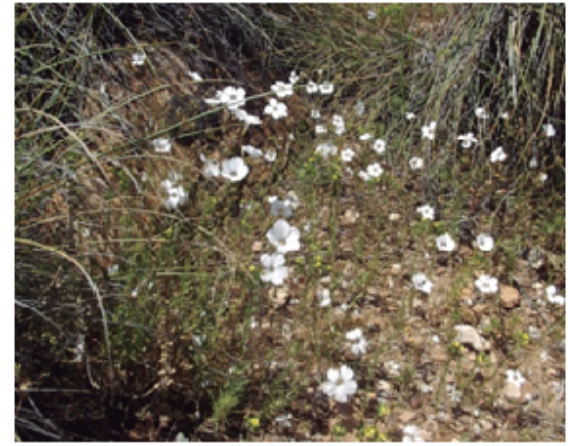

Linum tenue subsp. munbyanum

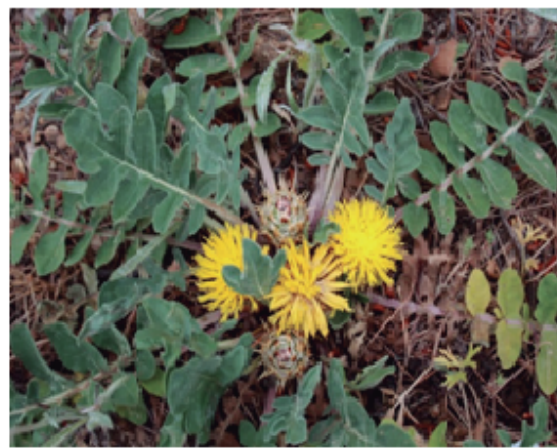

Centaurea oranensis

Planche 2. Quelques endémiques Algérie-Maroc de la région de Tiaret (Cliché: Miara M D.). Planche 2. Some endemic Algeria-Morocco of the region of Tiaret (Photo: Miara M D.).

Ce nombre peut être jugé comme assez intéressant pour une région relativement lointaine des frontières entre les deux pays comparé à ceux obtenus dans la région de Tlemcen par Benabadji et al. (2007) qui comptabilisent 4 espèces, de celui de Babali (2014) avec 6 espèces ou encore celui de Letreuch-Belarouci et al. (2009) qui signalent 7 espèces. Ces résultats semblent se justifier par le fait qu'il s'agit d'une région qui constitue une bonne partie de l'Atlas tellien occidental dont l'extension s'étend jusqu'au grand Atlas marocain.

Les taxons que nous avons identifiés restent globalement rares dans la région et la majorité d'entre eux n'ont été rencontrés qu'une seule foi. II s'agit de : Centaurea oranensis, Cistus munbyi, Cordylocarpus muricatus, Gagea algeriensis, Genista cephalantha, Linum tenue subsp. munbyanum, Romulea numidica, Salvia algeriensis, Sideritis guyoniana, Thymus munbyanus subsp.munbyanus et Verbascum faurei.

Les endemiques Algerie-Tunisie

Le nombre de taxons endémiques algéro- 


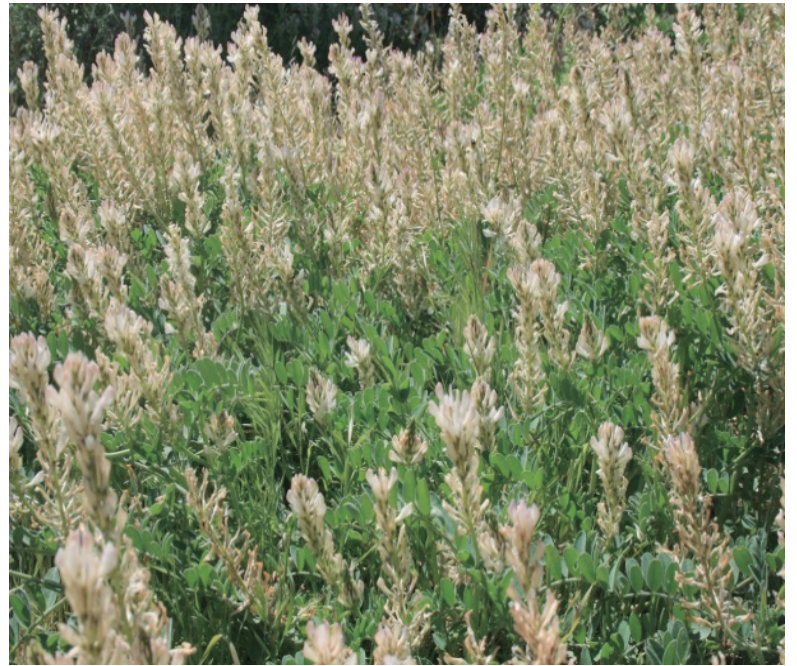

Hedysarum pallidum

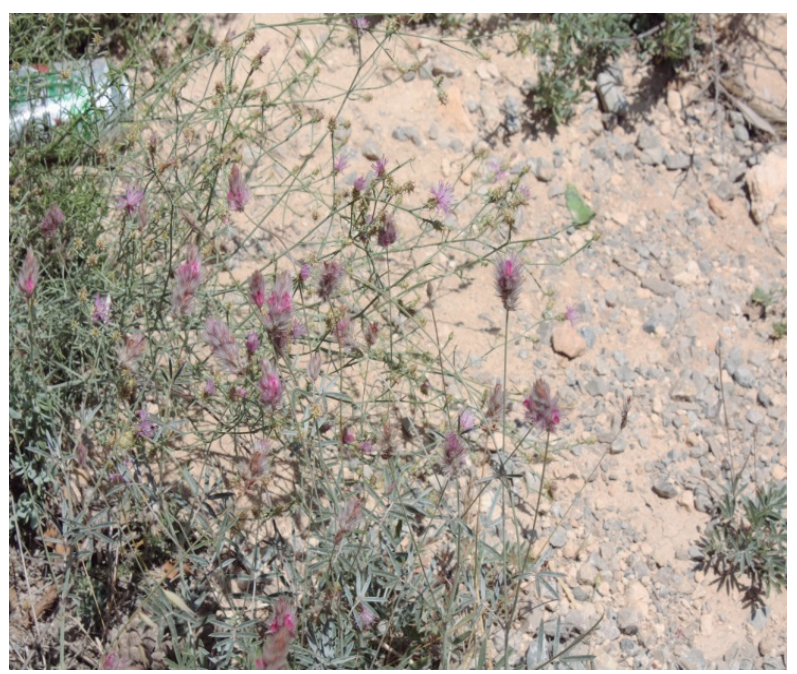

Ebenus pinnata

Planche 3. Quelques endémiques Afrique du Nord de la région de Tiaret (Cliché: Miara M D.). Planche 3. Some endemic North Africa of the region of Tiaret (Photo: Miara $M$ D.).

tunisiens reste faible (4 taxons) (Tab. 6), ce qui est évidemment logique pour une région qui se situe dans l'Atlas tellien occidental algérien, c'est-à-dire très loin des frontières Algéro-Tunisienne.

Ces taxons sont communs au niveau des deux pays avec des aires de répartition assez importants. Il s'agit de: Centaurea parviflora, Linum corymbiferum subsp. corymbiferum

Origanum vulgare subsp. glandulosum et Thymus pallescens.

Ce nombre de 4 taxons retrouvés représente près de $60 \%$ des taxons endémiques AlgérieTunisie signalés pour le secteur $\mathrm{O} 3$ qui sont du nombre de 7 taxons au total (Véla \& Benhouhou, 2007).

\section{Les endemiques nord-africaines}

Cet élément chorologique renferme la majorité des taxons endémiques avec 11 espèces (tab.7) dont certaines sont illustrées (planche 3).

II s'agit souvent de taxons rencontrés dans au moins trois pays de l'Afrique du Nord (Algérie, Maroc, Tunisie, Lybie) et s'étendant parfois jusqu'à l'Egypte.

Les espèces que nous avons identifiées sont : Asphodelus acaulis, Delphinium balansae, Ebenus pinnata, Genista tricuspidata subsp. tricuspidata, Hedysarum pallidum, Helianthemum helianthemoides, Helianthemum ledifolium subsp. apertum, Pancratium foetidum, Stipa balansae et Thymus algeriensis.

Medjahdi et al. (2009) signalent 17 taxons endémiques de l'Afrique du nord dans les monts de Traras alors que Babali (2014) en signale 11 pour les monts de Moutas. Comparé à ces observations, le nombre de 10 taxons retrouvés suite à la présente étude pourra être jugé important et assez intéressant.

\section{Les especes endemiques-rares}

Les espèces endémiques et rares ou plutôt «Trigger species» sensu Yahi et al. (2012) constituent selon ces auteurs un très bon outil d'évaluation et d'identification des zones de biodiversité importante «ZIP».

Ce concept qui réunit à la fois les critères de rareté et d'endémisme peut donner une idée assez satisfaisante sur l'importance d'une zone biogéographique donnée d'un point de vue de la richesse en espèces d'intérêt biologique et patrimoniale dans un cadre global de conservation et de développement durable.

Les espèces qui correspondent à ces critères au niveau de notre inventaire sont de l'ordre de 4

espèces à savoir: Centaurea phaeolepis, Otocarpus virgatus, Thymus lanceolatus, Thymus guyonii.

Ce nombre jugé assez important comparé à ceux des principales zones IPA d'Algérie (Yahi et al., 2012), permet de classer notre région parmi ces zones importantes pour les plantes.

D'après ces derniers auteurs, les espèces clés identifiées sur la base de la combinaison endémisme-rareté peuvent être utilisées pour caractériser l'intérêt particulier d'un site, devenant ainsi un outil utile à des faits de conservation.

\section{Les plantes rares (non-endemiques)}

La rareté a toujours provoqué la curiosité 


\begin{tabular}{|c|c|}
\hline Espèces rares & Biogéographie Q\&S (62) \\
\hline $\begin{array}{l}\text { Allium antiatlanticum Emb. \& Maire. } \\
=\text { Allium paniculatum var. rifanum }\end{array}$ & 03: Mts de Tlemcen. \\
\hline Althaea hirsuta L. & R: dans tout le tel \\
\hline Anarrhinum fruticosum Desf. & R: 03, HI, ASI. \\
\hline Anthemis arvensis $\mathrm{L}$. & R: Al: env. d'Alger, K3: basse Seybouse \\
\hline $\begin{array}{l}\text { Aphanes microcarpa (Boiss. \& Reut.) Rothm. } \\
=\text { Alchemilla arvensis ssp. microcarpa }\end{array}$ & RR: Tiaret, Tlemcen \\
\hline Arabidopsis thaliana (L.) Heynh. = Sysimbrium thalianum & C: dans le Tell algéro-constantinois. R: en Oranie \\
\hline $\begin{array}{l}\text { Arenaria leptoclados (Rchb.) Guss. } \\
=\text { Arenaria serpyllifolia ssp. leptoclados. }\end{array}$ & $\mathrm{RR}: \mathrm{A} 2$ \\
\hline Astragalus alopecuroides L. = Astragalus narbonensis & R: Aurès, Biban, Ouarsenis, Steppes d'alfa du Sud oranais \\
\hline Bupleurum rigidum $\mathrm{L}$. & AR: HI-2, ASI-2-3, RR: ailleurs \\
\hline Cataposium salzmanii & RR: K2: Stora, 03: entre Mascara et Saïda \\
\hline Crocus nevadensis Amo \& Campo & R: 03: Mts de Tlemcen et de Daya, $\mathrm{H} 1$ \\
\hline Cuminum cyminum L. & $\mathrm{R}: \mathrm{HI}, \mathrm{AS} 1, \mathrm{H} 2$ \\
\hline $\begin{array}{l}\text { Cytinus hypocistis subsp. macranthus Wettst. } \\
=\text { Cytinus hypocistis ssp.lutescens }\end{array}$ & RR : sur Fumana, Helianthemum, Halimium, Sidi-Ferruch, Oran. \\
\hline $\begin{array}{l}\text { Eremopyrum buonaepartis (Spreng.) Nevski. } \\
=\text { Agropyron buonapartis }\end{array}$ & Oranie. R: HI \\
\hline Euphorbia squamigera Loisel. & R: 01-3 \\
\hline Galium setaceum Lam. & AR: H2, AS3, RR ailleurs \\
\hline Galium verticillatum Danthoine & R: 03, HI-2, AS, RR ailleurs: KI: Djurdjura \\
\hline Geranium rotundifolium $\mathrm{L}$. & R: çà et là en Algérie, surtout en montagne \\
\hline Medicago littoralis Loisel. & AC: HI-2, ASI-2-3, SS. R: dans le Tell \\
\hline Medicago orbicularis (L.) Bartal. & C: dans le Tell. R: H1-2. AS1-2-3 \\
\hline Micromeria nervosa (Desf.) Benth. & RR: Al: de Ténès à Cherchell \\
\hline Minuartia campestris & $\mathrm{R}: \mathrm{O3}$ \\
\hline Myosotis discolor Pers. = Myosotis versicolor & R: Al, KI-2-3. RR: ailleurs: Tiaret \\
\hline $\begin{array}{l}\text { Narduroides salzmannii (Boiss.) Rouy. } \\
=\text { Catapodium salzmanni }\end{array}$ & RR: K2: Stora, 03 \\
\hline Neotinea intacta & R: çà et là dans le Tell littoral \\
\hline Orchis longicornu & AC dans le Tel, $R$ en Oranie \\
\hline $\begin{array}{l}\text { Phlomis herba-venti subsp. pungens (Willd.) } \\
\text { Maire ex DeFilipps }\end{array}$ & R: çà et là dans toute l'Algérie \\
\hline Rochelia disperma (L. f.) C. Koch & CC: H, AS. R: dans le Nord : Cl, 03 \\
\hline Salvia argentea & C: HI-2 \\
\hline Scorpiurus muricatus L. = Scorpiurus muricatus ssp. sub-villosus & $\mathrm{R}: \mathrm{Cl}-\mathrm{H} 2$. \\
\hline Scorpiurus muricatus L. subsp. muricatus & RR: Cap Aokas, Cherchell. \\
\hline Silene cerastoides $\mathrm{L}$. & R: Al, 01. RR: HI: Aumale \\
\hline Silene coelirosa (L.) Godr. & C: Tell-algéro-constantinois. R: en Oranie \\
\hline Silene secundiflora Otth & R: çà et là dans toute l'Algérie \\
\hline Verbascum blattaria L. & $\mathrm{R}: \mathrm{A} 2, \mathrm{KI} 2-3,03, \mathrm{Cl}, \mathrm{ASI}-3$ \\
\hline Withania frutescens (L.) Pauquy & CC 01-2 \\
\hline
\end{tabular}

Table 8. Les taxons rares retrouvés dans la région de Tiaret. Table 8. Rare taxa found in the region of Tiaret. 


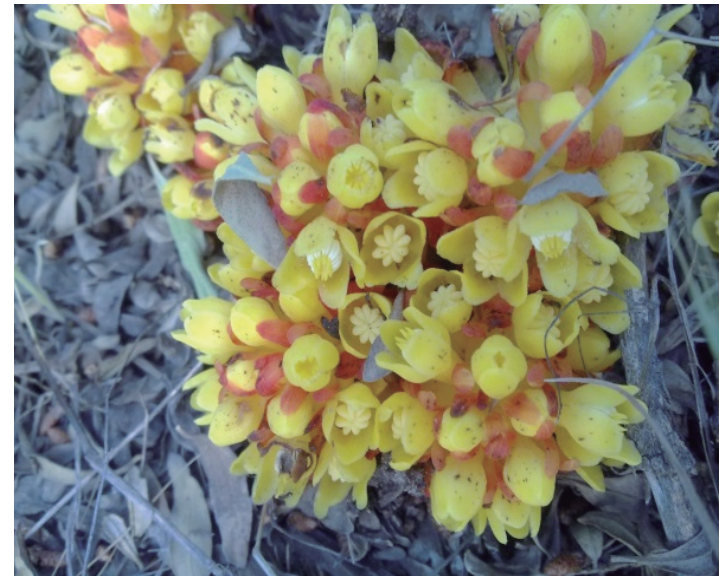

Cytinus hypocistis subsp.

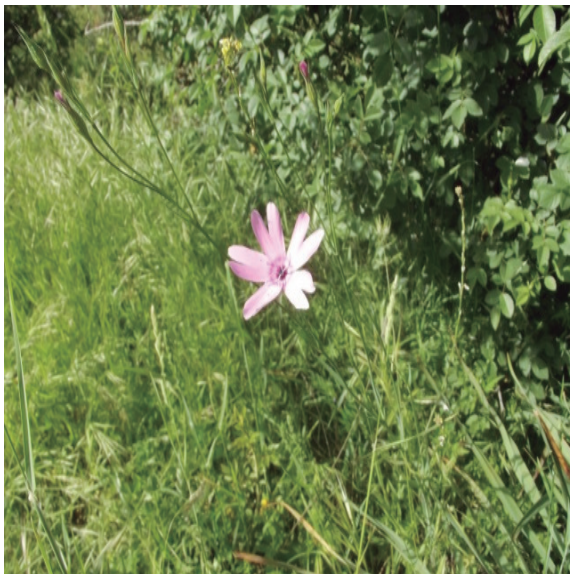

Silene coelirosa

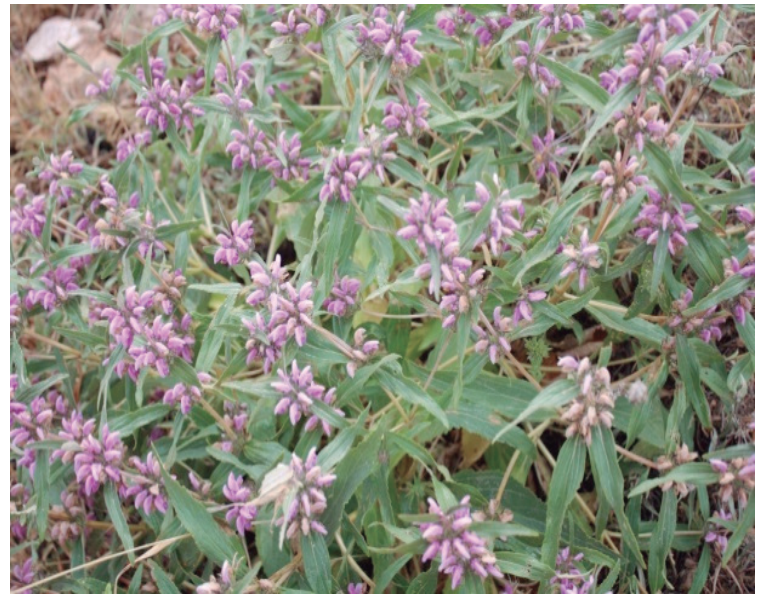

Phlomis herba-venti subsp. pungens

Planche 4. Quelques plantes rares de la région de Tiaret (Cliché: Miara M D.). Planche 4. Some rare plants from the region of Tiaret (Photo: Miara $M$ D.).

et la convoitise des hommes. De tout temps, collectionneurs comme naturalistes sont attirés par des pièces, des timbres de collection, rares ou originaux, tout comme par des espèces encore non décrites, en particulier si elles sont inféodées à une zone géographique restreinte (Hamel et al., 2013).

L'analyse de l'abondance des especes selon (Quézel \& Santa, 1962-63) a permis de mettre en exergue 36 taxons rares $(R)$ ou très rares (RR), pour la région de Tiaret, le secteur biogéographique $\mathrm{O} 3$ ou bien pour le pays (Tab. 8). Or, Nous n'avons pas tenu compte de ceux figurant comme assez rares (AR). Ces proportions de rareté correspondent à $12 \%$ des espèces rares du secteur 03 d'après Véla \& Benhouhou (2007).

Les plantes rares ont donc une grande valeur en terme de conservation, soit pour des raisons patrimoniales, soit pour leur risque d'extinction (Pimm et al., 1988; Gaston et al., 1991).

Ces espèces, dont certaines sont illustrées (planche 4), bien qu'elles puissent être rares ou très rares au niveau régional, leur existence et abondance au niveau des autres régions du pays n'est pas forcément de même. Toutefois, nous insistons sur leur valeur à cette échelle régionale comme des taxons à préserver.

Sur le plan pratique, les indices de rareté régionale ou nationale contenus dans la flore de Quézel \& Santa (1962-63) doivent être actualisés à la lumière des nouvelles données.

\section{Les especes protegees}

Nos recherches nous ont conduits à identifier plusieurs taxons figurant dans la liste des espèces 


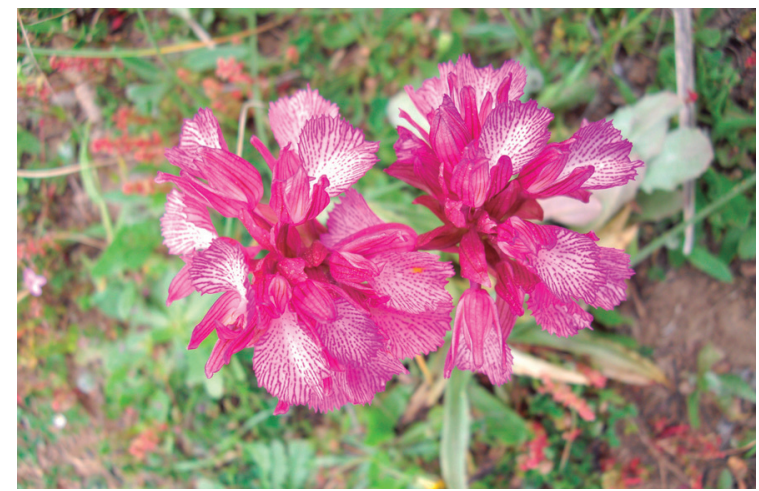

Orchis papilionacea

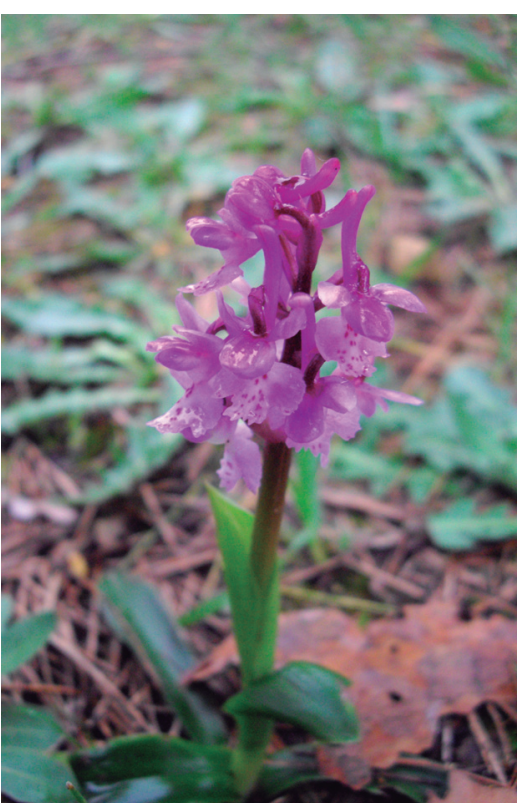

Orchis mascula ssp. olbiensis

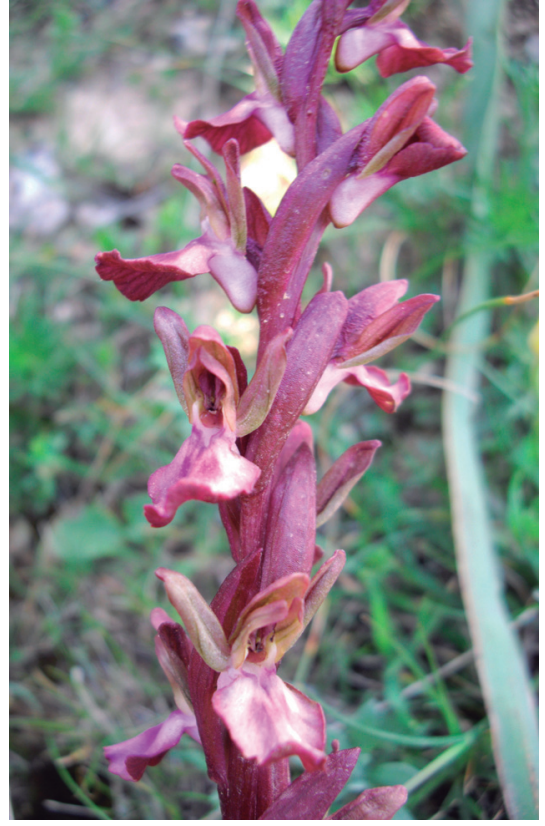

Orchis collina

Planche 5. Quelques plantes menacées de la région de Tiaret (Cliché: Ait Hammou M.). Planche 5. Some threatened plants of the region of Tiaret (Photo: Ait Hammou M.).

protégées par la loi algérienne (décret exécutif $\mathrm{n}^{\circ} 12-03$ du 10 safar 1433 correspondant au 4 janvier 2012 fixant la liste des espèces végétales non cultivées protégées).

Ces plantes ont bénéficié d'un statut légal de plantes protégées car elles sont jugées vulnérables et menacées. II s'agit de 17 taxons comptant plusieurs Orchidées (planche 5) à savoir: Adenocarpus faurei, Celsia faurei, Centaurea phaeolepis, Gagea algeriensis, Helianthemum helianthemoides, Juniperus oxycedrus, Orchis collina, Orchis coriophora ssp. fragrans, Orchis longicornu, Orchis mascula ssp. olbiensis, Orchis papillionacea, Orchis tridentata, Otocarpus virgatus, Tetraclinis articulata, Thymus guyonii et Thymus lanceolatus.
Dans ce contexte, la recherche de quelques taxons endémiques et rares à aire biogéographique restreinte n'a pas toujours été fructueuse. En effet, certains taxons risquent fortement l'extinction vue leur localisation, et les conditions anthropiques défavorables pour leur survie.

D'après (May et al., 1995), le rythme actuel d'extinction des espèces serait 100 à 1000 fois supérieur aux rythmes déduits de données paléontologiques.

Cela pourrait être le cas d'Adenocarpus faurei, Trisetaria nitida ou encore Centaurea obtusiloba. Ces endémiques algériennes très rares et localisées qui n'ont pas été revues depuis leurs descriptions à l'époque coloniale. En effet, la flore du bassin méditerranéen est aujourd'hui très sérieusement 
menacée, en raison de la forte régression des milieux naturels sous l'action de l'homme, mais aussi parce que cette région serait l'une des plus exposées aux changements climatiques globaux (Sala et al., 2000; Médail \& Quézel, 2003).

\section{Conclusion}

Cette analyse apporte plusieurs informations pertinentes sur la flore endémique, rare et menacée de l'Atlas tellien occidental de Tiaret.

Le nombre important des plantes endémiques et rares que nous avons identifiés permet de proposer le secteur Atlasique de Tiaret comme une zone importante pour les plantes (ZIP).

L'analyse taxonomique et biogéographique des données récoltées sur le terrain a permis d'abord de renforcer et compléter les connaissances sur la flore régionale et d'apporter plus

d'informations sur le plan de la distribution géographique de certains taxons.

Globalement, cette analyse nous informe sur un état préoccupant de notre flore notamment celle endémique, car plusieurs taxons n'ont été rencontrée qu'une seule fois, alors que d'autres ont probablement disparu.

C'est ainsi que nous appelons en urgence a une meilleure considération de ces espaces naturels qui abritent ce patrimoine biologique unique par ses valeurs patrimoniales et biologiques, mais aussi par sa diversité floristique regorgée d'une panoplie de belles fleurs méditerranéennes. La protection et la conservation de ces régions demeurent aujourd'hui une priorité à cause de la dégradation des habitats naturels au niveau des massifs forestiers et préforestiers. Cela va contribuer certainement a plus de raréfaction voir à la disparition progressive des plantes endémiques et rares souvent vulnérables face aux contraintes écologiques (aridité climatique) et anthropiques (pâturage extensif, feux,...etc).

\section{Remerciements}

Les auteurs tiennent à remercier Dr. Errol Véla (Université de Montpellier 2) pour son aide à l'identification des spécimens d'herbier ainsi que pour ses conseils et orientations.

\section{Références}

Babali, B. (2014). Contribution à une étude phytoécologique des monts de Moutas (TlemcenAlgérie occidentale): Aspects syntaxonomique, biogéographique etdynamique.Thése Doct. Univ Tlemcen. 160p.
Battandier, J.A. \& Trabut, L.C. $(1888,1890)$. Flore d'Algérie. Adolphe Jourdan éd., Alger, 825 p.

Battandier, J.A. \& Trabut, L.C. (1895). Monocotylédones (Flore de l'Algérie). Typographie Adolphe Jourdan, Alger, $256 \mathrm{p}$.

Benabadji, N., Benmansour, D. \& Bouazza, M. (2007). La flore des monts d'Ain fezza dans l'ouest algerien, biodiversite et dynamique. Sciences \& Technologie. N²6, pp.47-59.

Dobignard, A. \& Chatelain, C. $(2010,2011,2012$, et 2013). Index synonymique de la Flore d'Afrique du Nord. Conservatoire et Jardin botaniques de la Ville de Genève (CH), Vol. 1, 2, 3, 4 et 5.

Duvignaud, P. (1992). Aménagement et gestion du territoire. Application en Algérie (région de Tiaret et Alger). Univ de Nice-Sophia Antipolis. 253p.

El Oualidi, J., Khamar, H., Fennane, M., Ibn Tattou, M., Chauvet, S. \& Taleb, M.S. (2012). Checklist des endémiques et spécimens types de la flore vasculaire de l'Afrique du Nord. Doc Inst Sci. Universite

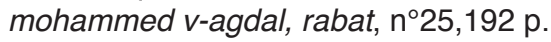

Gaston, K. J. (1991). How large is species geographical range?.Oikos, Vol. 61, 434-438.

Guittonneau, G.G. (1972). Contribution à l'étude biosystématique du genre Erodium L'Hér. dans le bassin méditerranéen occidental. Boissiera, 20: 89.

Hamel, T., Seridi, R., De Belair, G., Slimani, A. \& Babali, B. (2013). Flore vasculaire rare et endémique de la péninsule de l'Edough (Nord-Est algérien). Rev. Sci. Technol., Synthèse. 26, $65-74$.

Letreuch-Belarouci, A., Medjahdi, B., Letreuch-Belarouci, N. \& Benabdeli, K. (2009). Diversité floristique des subéraies du parc national de Tlemcen (Algérie). Act. Bot. Malacitana, 34, 77-89.

Maire, R. (1952, 1987). Flore de l'Afrique du Nord (Maroc, Algérie, Tunisie, Tripolitaine, Cyrénaïque et Sahara). Paris : éditions Le Chevalier ; 16 vol. Parus.

Maire, R. (1965). Flore de l'Afrique du Nord (Maroc, Algérie, Tunisie, Tripolitaine, Cyrénaïque et Sahara), vol. XII : Rhoeadales : Papaveraceae, sf. Fumarioidea p.p. ; Capparidaceae; Cruciferae p.p. Le Chevalier édit. Paris, $407 \mathrm{p}$.

MATE, (Ministère de l'aménagement du territoire et de l'environnement). (2014) $5^{\text {ème }}$ rapport national sur la mise en oeuvre de la convention sur la diversite biologique au niveau national. 128p.

Médail, F. \& Quézel, P. (2003). Conséquences écologiques possibles des changements climatiques sur la flore et la végétation du bassinméditerranéen. Bocconea, 16(1).

Medjahdi, B., Ibn Tattou, M., Barkat, D. \& Benabedli, K. (2009). La flore vasculaire des Monts des Traras (Nord Ouest Algérien). Acta Bot. Malacitana, 34, 57-75.

Miara, M.D., Ait Hammou, M., Hadjadj-Aoul, S. \& Hamerlain, A.S. (2013). Bioclimats, étages de végétation et zonation altitudinale des groupements végétaux dans la région de Tiaret. Rev. Ecol. Env. 9 (7 p.). En ligne : fsnv.univ-tiaret.dz/miara_article.pdf.

Miara M.D., Ait Hammou, M. \& Hadjadj-Aoul, S. (2014). Redécouverte d'Otocarpus virgatus Durieu (Brassicasseae) Dans la région de Tiaret (N-O Algérie). Bul. Soc. Lin. Prov. France.T (65) : 31-35p. 
Miara, M.D. (2017). Analyse floristique et structure de la végétation de la région de Tiaret. Thése Doct. Univ Oran. 162p.

Miara, M.D., Ait Hammou, M. \& Skipper, J. (2018). The disappearance of Adenocarpus faurei Maire (Fabaceae-Fabales) in Algeria, an another loss for world biodiversity. Journal of Threatened taxa. Soumis.

Myers, N. (1990). The biodiversity challenge: Expanded hotspotanalysis. Environmentalist, 10, 243-256.

Myers, N. (2003). Biodiversity hotspot revisited. Bioscience, 53, 916-917.

Pimm, S.L., Jones, H.L. \& Diamond, J. (1988). On the risk of extinction. The American Naturalist, Vol. 132 , 757-785.

Quézel, P. \& Santa, S. (1962). Nouvelle flore de l'Algérie et des régions désertiques méridionales. CNRS. Paris. 2 vols. $1170 \mathrm{p}$.

Radford, E.A., Catullo, G. \& Montmollin, B. (2011). Zones importantes pour les plantes en Mediterranee meridionale et orientate, sites prioritaires pour la conservation. UICN.Plantilife International. 134p.

Sala, O.E., Chapin, F.S.I., Armesto, J.J., Berlow, E., Bloomfield, J., Dirzo, R., Huber-Sanwald, E., Huenneke, L.F., Jackson, R.B., Kinzig, A., Leemans, R., Lodge, D.M., Mooney, H.A., Oesterheld, M., Poff, N.L., Sykes, M.T., Walker, B.H., Walker, M. \& Wall, D.H (2000). Global biodiversity scenarios for the year 2000.Science, Vol. 287, 1 770-1 774.

Trabut, L.C. (1887). D'Oran à Mechria, notes botaniques et catalogue des plantes remarquables. Adolphe Jourdan édit., Alger, 36p.

Véla, E. \& Benhouhou, S. (2007). Evaluation d'un nouveau point chaud de biodiversité végétale dans le bassin méditerranéen (Afrique du nord). C.R. Biologies ; 330 : 589-605.

Yahi, N., Véla, E., Benhouhou, S., De Belair, G. \& Gharzouli, R. (2012). Identifying Important Plants Areas (Key Biodiversity Areas for Plants) in northern Algeria.Journal of Threatened Taxa, 4(8), 2753-2765. 